\title{
Analysis of Cultured Neuronal Networks Using Intraburst Firing Characteristics
}

\author{
Jan Stegenga*, Joost Le Feber, Enrico Marani, and Wim L. C. Rutten
}

\begin{abstract}
It is an open question whether neuronal networks, cultured on multielectrode arrays, retain any capability to usefully process information (learning and memory). A necessary prerequisite for learning is that stimulation can induce lasting changes in the network. To observe these changes, one needs a method to describe the network in sufficient detail, while stable in normal circumstances. We analyzed the spontaneous bursting activity that is encountered in dissociated cultures of rat neocortical cells. Burst profiles (BPs) were made by estimating the instantaneous array-wide firing frequency. The shape of the BPs was found to be stable on a time scale of hours. Spatiotemporal detail is provided by analyzing the instantaneous firing frequency per electrode. The resulting phase profiles (PPs) were estimated by aligning BPs to their peak spiking rate over a period of $15 \mathrm{~min}$. The PPs reveal a stable spatiotemporal pattern of activity during bursts over a period of several hours, making them useful for plasticity and learning studies. We also show that PPs can be used to estimate conditional firing probabilities. Doing so, yields an approach in which network bursting behavior and functional connectivity can be studied.
\end{abstract}

Index Terms-Cultured neuronal networks, functional connectivity, multielectrode arrays (MEAs), network bursts, spontaneous activity.

\section{INTRODUCTION}

$\mathbf{T}$ HE culturing of neurons on microelectrode arrays (MEAs) offers the possibility to study the patterns of action potentials generated by a relatively small, single-layered networks of neurons. It has been shown that individual cultured neurons retain many properties of their in vivo counterparts [1], [2], it is hoped that some properties of networks of neurons are also retained. Learning and memory are two key properties of a neuronal network. There are several studies that suggest a learning behavior in networks cultured on MEAs [3]-[7], others speak of induced plasticity [8]-[12]. The difference is that one can only speak of learning when some sort of improvement in the

Manuscript received April 18, 2007; revised September 18, 2007. Asterisk indicates corresponding author.

*J. Stegenga is with the Institute of Biomedical Technology (BMTI), Department of Electrical engineering Electrical Engineering, Mathematics and Computer Science (EEMCS), Biomedical signals and systems group (BSS), University of Twente, Enschede 7500 AE, The Netherlands P.O. Box 2177500 AE Enschede The Netherlands (e-mail: j.stegenga@utwente.nl).

J. Le Feber and W. L. C. Rutten are with the Institute of Biomedical Technology (BMTI), University of Twente, Enschede 7500 AE, The Netherlands.

E. Marani is with the Institute of Biomedical Technology (BMTI), University of Twente, Enschede $7500 \mathrm{AE}$, The Netherlands and also with the Department of Neurosurgery, Leiden University Medical Center, Leiden 2300 RC, The Netherlands.

Color versions of one or more of the figures in this paper are available online at http://ieeexplore.iee.org.

Digital Object Identifier 10.1109/TBME.2007.913987 response can be observed (i.e., goal-directed behavior), while plasticity merely requires the observation of changes induced by stimulation. Most studies use a stimulus-response test before and after the experiment in order to reveal the changes in a network after applying their learning or plasticity inducing algorithms [3], [4], [6], [7], [11]. These stimulus-response tests are relatively easy to conduct and can be repeated several times to enable statistical evaluation. However, it has been shown that responses to stimuli change over time [12]. This limits the number of responses that are allowed to be averaged and (therefore) also the amount of detail that can be extracted from these responses. In fact, the changing of responses suggests that the test stimuli themselves are inducing changes in the network.

Several groups focus their attention to changes that may occur in the spontaneous activity before and after an experiment [10], [12], [13]. In order to distinguish changes in activity after learning experiments from the activity before, normal development should be known. In this view, it is important to know the timescale at which the chosen parameters change during normal development, because the spontaneous activity can change over several timescales (from minutes to days). The parameters should be stable over the duration of an experiment (usually several hours) to be useful for analyzing changes induced by stimulation, yet be sensitive enough to pick up the induced changes.

Spontaneous activity is dominated by network bursts-periods in which the spiking activity is very high compared to the nominal level. Bursts are present throughout the cultures' measured lifetime, starting at four to seven days in vitro (DIV) and lasting for the entire culturing period [14], [15]. The appearance and structure of bursts change with age [14], [16]-[20]. Due to the fact that the spatiotemporal structure of bursts changes with age but appears to be quite stable over a period of hours [3], [19], parameters extracted from bursts should have a natural timescale of no change during which it is possible to observe changes due to stimulation algorithms. In a detailed study, Van Pelt et al. [19] reported on the bursting behavior during the development of cultures (from 7 DIV to 49 DIV). They show that bursts change significantly during development by analyzing the array-wide spiking rate (AWSR) in intervals of $10 \mathrm{~ms}$. The AWSR is a summation of action potentials over all electrodes. Their main finding was that the AWSR during bursts has long rise and long fall times in early development, which changes to very sharp and intense profiles after about 25 DIV [19, Fig. 9]. They also show that activity during bursts is electrode specific, such that neurons have a preferred phase during which they are most active. These and other changes in AWSR during development coincide with changes in synaptic connections [8], [21]-[28], suggesting that network bursts carry infor- 
mation about the networks' connectivity. Provided that network bursts are selectively sensitive to (changes in) synaptic a network state for experiments that aim to induce synaptic changes can be derived from them.

Network bursts parameters have already been used in various studies involving plasticity. In 1998, Meada et al. [8] reported an effect after tetanisation (stimulation in trains with an interstimulation interval of $50 \mathrm{~ms}$, lasting $1 \mathrm{~s}$ ) in burst frequency and the number of spikes in bursts. However, the effect was observed for only $20 \mathrm{~min}$ (due to recording length). Also, changes in these coarse parameters require a large change in network connectivity because they are unlikely to change when a limited number of connections is altered. Indeed, Wagenaar et al. [9] carried out similar experiments and found no significant changes in either responses to test stimuli or in spontaneous bursts (burst frequency and AWSR rise and fall times).

\section{A. Time Development and Composition of Network Bursts}

The analysis presented in this paper starts by considering the AWSR during network bursts in detail. A burst profile (BP) was calculated by smoothing the train of action potentials by Gaussian filtering. The BPs were examined over a period of several days to show that bursts change their morphology and reveal short-term stability. Next, the network bursts were analyzed in the spatiotemporal domain (i.e., per recording site). The rationale for doing so was the limited amount of detail that the BPs offer because they were calculated from the AWSR, which includes only temporal information. The obtained electrode-specific profiles were called phase profiles (PPs) because they show the electrode's contribution at times relative to the time of maximum network synchrony. It has been suggested in several studies that there is an order in which sites become active during bursts. Beggs and Plenz [29] classified network bursts in coronal slices of rat cortex using the temporal order of firing. They showed that multiple spatiotemporal burst patterns are present at any time and suggest that these patterns form the basis for memory. Using a different approach, Baruchi and Ben-Jacob [30] showed that dissociated cultures of cortical neurons exhibit network bursts with a very similar structure. A spatiotemporal pattern during network bursts was also observed by Van Pelt et al. [19], even though no attempt was made to classify the bursts. The latter result may indicate that there is a dominant pattern present. In this study, we first confirmed the presence of a dominant pattern, and then calculated PPs by aligning several bursts without classification. PPs describe network bursting activity and, thus, form an indirect measure of the synaptic connectivity. In support of this view, we show that PPs can be used to estimate conditional firing probabilities (CFPs). The functional connectivity found by methods, such as cross-correlation analysis [31] and conditional firing probabilities [32] in terms of strength and latency, can thus be connected to PPs. How PPs and CFPs are mathematically related is described in the Appendix.

\section{MATERIALS AND METHODS}

\section{A. Cell Cultures}

Cortical neurons were obtained from either newborn or E18 Wistar rats by trituration and chemical dissociation using

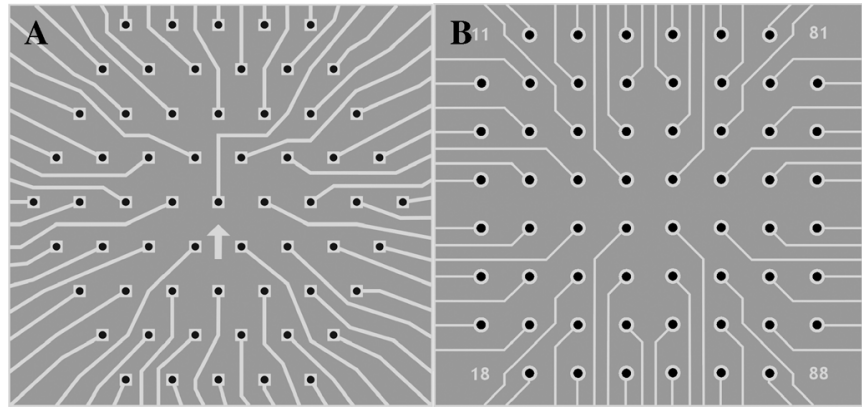

Fig. 1. (a) Hexagonal layout of an UTMEA. electrode diameter: $12 \mu \mathrm{m}$; spacing: $80 \mu \mathrm{m}$. (b) Square layout of an MCSMEA. Electrode diameter: 10 $\mu \mathrm{m}$; spacing: $100 \mu \mathrm{m}$

trypsin. The cells were plated at a concentration of $10^{6}$ cells $/ \mathrm{ml}$ (Romijn's R12 medium [33]) and allowed to adhere for $2 \mathrm{~h}$. MEAs were coated with polyethylene-imine (PEI) to increase adhesion. The nonadhering cells were removed by refreshing medium, and $600 \mu \mathrm{l}$ of R12 medium was added. The resulting monolayer had a density of about 5000 cells $/ \mathrm{mm}^{2}$. The medium was entirely changed twice per week. The cultures were stored in an incubator at $37^{\circ} \mathrm{C}$, at a $\mathrm{CO}_{2}$ concentration of $5 \%$ and near $100 \%$ humidity. During measurements, the cultures were covered with a lid and tightly sealed with parafilm. Cultures were allowed to settle for $20 \mathrm{~min}$ before the start of measurement.

\section{B. Measurement Setup}

60-Channel Recordings: We used the MC1060BC preamplifier and FA60s filter amplifier (both MultiChannelSystems $\mathrm{GmbH}$, Reutlingen, Germany) to prepare the signals for analog-to-digital conversion. Amplification was 1000 times in a range from $100 \mathrm{~Hz}$ to $6000 \mathrm{~Hz}$. A 6024E data-acquisition card (National Instruments, Austin, TX) was used to record all 60 channels at $16 \mathrm{kHz}$. Custom-made Labview (National Instruments, Austin, TX) programs were used to control data acquisition and applied a threshold detection scheme with the objective of data reduction. The actual detection of action potentials was performed offline. During the experiments, the temperature was controlled at $36.0^{\circ} \mathrm{C}$, using a TC01 (multichannel systems) temperature controller. Noise levels were typically 3 to $5 \mu \mathrm{V}_{\mathrm{RMS}}$. The MEAs had 60 titanium-nitride electrodes of $10 \mu \mathrm{m}$ in diameter, spaced $100 \mu \mathrm{m}$ apart in a square grid [see Fig. 1(b)].

Recordings before June 1, 2005, were made using MEAs manufactured at the University of Twente (referred to as UTMEAs). These had 61 gold electrodes and were $12 \mu \mathrm{m}$ in diameter and spaced $80 \mu \mathrm{m}$ apart in a hexagonal configuration [Fig. 1(a)]. After coating with platinum black, the noise levels were typically $7 \mu \mathrm{V}_{\text {RMS }}$. The area surrounding the electrode area was coated with Silastic 734, which increased the useful lifespan of the MEAs. The setup used a custom-made 16-channel amplifier, with an amplification of 230 in a range of $300 \mathrm{~Hz}$ to $6 \mathrm{kHz}$. A PCI-6023E data-acquisition card (National Instruments, Austin, TX) sampled the signals at $12500 \mathrm{~Hz}$. The temperature was controlled at $36.0^{\circ} \mathrm{C}$. 
TABLE I

OVERVIEW OF THE DATASET IN TERMS OF MEA TYPE, NUMBER OF MEASurements, AND Number of CULTURES. THE Number oF PREPARATIONS OF 1 DAY-Old RATS AND THE NUMBER OF E18 PREPARATIONS IS GIVEN BETWEEN THE PARENTHESES

\begin{tabular}{|l|l|l|l|l|}
\cline { 2 - 5 } \multicolumn{1}{c|}{} & \multicolumn{2}{l|}{ UTMEA } & \multicolumn{2}{l|}{ MCSMEA } \\
\cline { 2 - 5 } \multicolumn{1}{c|}{} & sessions & cultures & sessions & cultures \\
\hline Single day & 3 & $3(3 / 0)$ & 25 & $9(9 / 0)$ \\
\hline Multiple day & - & - & 41 & $4(3 / 1)$ \\
\hline
\end{tabular}

\section{Experiments}

The experiments can be subdivided into two categories: 1 ) single-day measurement sessions, typically lasting $2 \mathrm{~h}$, used to assess stability on a timescale of minutes to hours and 2) sets of measurements (i.e. multiple days), used to assess developmental changes as well as stability. Each set of measurements consists of at least five measurement sessions of a single culture (Table I).

\section{Data Processing}

Online preprocessing consisted of detecting threshold crossings and storing $10 \mathrm{~ms}$ of data for each candidate action potential. The threshold level was set at 5.5 times the estimated rms noise level of the electrode, which was continuously monitored. Detections were validated offline using the algorithm described in [34], as this was found to be a fast and reliable method. We required that no more than four detections occurred on the same sample in order to suppress synchronous artefacts picked up from external sources. In a heavily bursting culture, this requirement resulted in less than $0.1 \%$ data loss, while still being effective in detecting and removing these artifacts.

We refer to bursts as network bursts when the total firing rate, as determined in 10-ms bins, exceeded a threshold. The threshold was set at two spikes for each electrode that was considered active (spike rate $>0.1 \mathrm{~Hz}$ ). Whenever a bin exceeded the threshold, a BP was calculated in order to estimate the time at which the peak AWSR occurred $\left(t_{c}\right)$. Once found, BPs and PPs were calculated from $t_{c}-300 \mathrm{~ms}$ to $t_{c}+300 \mathrm{~ms}$. Threshold crossings were treated in order of size, and overlap between profiles was prevented by setting all bins in $t_{c}-600$ to $t_{c}+600 \mathrm{~ms}$ to zero. The influence of bin width and threshold combinations were tested, but led to comparable results between natural limits of: 1) high threshold-small bin width combinations that lead to missed events and 2) a low threshold-large bin width combinations that lead to false detections, and 3) bin widths that exceed interburst intervals.

$\mathrm{A} \mathrm{BP}$ is an estimation of the instantaneous array-wide spiking rate (AWSR). To this end, all spike occurrences in a burst are taken together and convolved with a Gaussian probability density function (Fig. 2), with a standard deviation (SD) of $5 \mathrm{~ms}$. This was wide enough to provide a smooth result near the maximum AWSR, and small enough so as not to obscure important details of the AWSR. A smooth graph near the maximum AWSR was important for aligning and comparing profiles. The profiles were $600 \mathrm{~ms}$ wide, large enough to capture the relevant features in most cases. Exceptions to the above were very young cultures in which bursts were typically longer and the maximum AWSR was lower. In these cases, a wider SD would have led to better

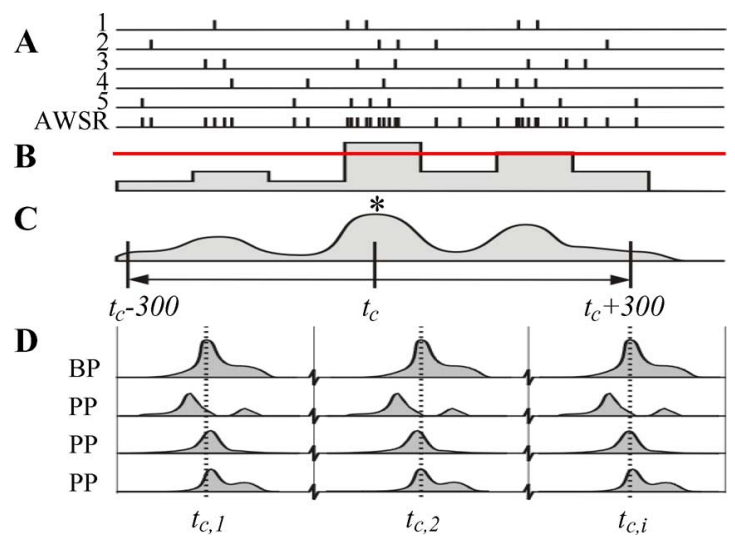

Fig. 2. Calculation of BPs. (a) Action potentials across all electrodes are added to make the AWSR. (b) The AWSR is binned and compared with a threshold. (c) Whenever the number of spikes per bin crosses a threshold, a profile is calculated by convoluting the AWSR spike train with a Gaussian (* denotes the burst peak). (d) The dataset is a series of BPs and phase profiles (PP).

results, but a changing SD would make comparing profiles with each other less direct; therefore, we used a fixed SD and a fixed profile width.

The number of spikes that were recorded per electrode per burst was relatively small, when compared to the number of spikes in the AWSR. Therefore, more averaging was required to obtain a useful estimation of PPs. Averaging over multiple bursts, aligned to their peak AWSR was found to yield stable PPs. Averaging over a small amount of time (i.e., $15 \mathrm{~min}$ ) was justified by the results of cluster analysis (as will be seen).

We used the correlation coefficient to quantify changes between profiles. For two discrete time BPs, $A=\left\{a_{1}, a_{2}, \ldots a_{K}\right\}$ and $B=\left\{b_{1}, b_{2}, \ldots b_{K}\right\}$, the correlation coefficient is defined as

$$
\begin{aligned}
C(A, B) & =\frac{\sigma_{A B}}{\sigma_{A} \sigma_{B}} \\
& =\frac{\sum\left(a_{k}-\mu_{A}\right)\left(b_{k}-\mu_{B}\right)}{\left(\sum\left(a_{k}-\mu_{A}\right)^{2}\right)^{0.5}\left(\sum\left(b_{k}-\mu_{B}\right)^{2}\right)^{0.5}}
\end{aligned}
$$

where the summations are over the discrete time parameter $k$, and $\mu$ is the average firing rate within one burst. The correlation coefficient is sensitive to changes in shape, but insensitive to changes in magnitude (i.e., total number of spikes per burst).

Relationships between single-electrode firing patterns were calculated using (9) from the Appendix

$$
\mathrm{CFP}_{i j}(\tau)=J \int_{t_{m}} f_{i}\left(t_{m}-\tau\right) f_{j}\left(t_{m}\right) d t_{m}
$$

where $f_{i}(t)$ represents the PP of electrode $i$, scaled to unit area and $J$ is a firing rate-dependent scaling factor. As is explained in the Appendix, the outcome of convolving phase profiles yields an estimation of the conditional firing probability (CFP).

We have used the algorithm described by Segev et al. [35] to check whether there are discernible differences between bursts. Shortly, the distance (i.e., dissimilarity) between bursts was calculated based on cross correlations between electrode firing patterns. The dendrogram clustering method used these distances to assign bursts to one of five classes. 

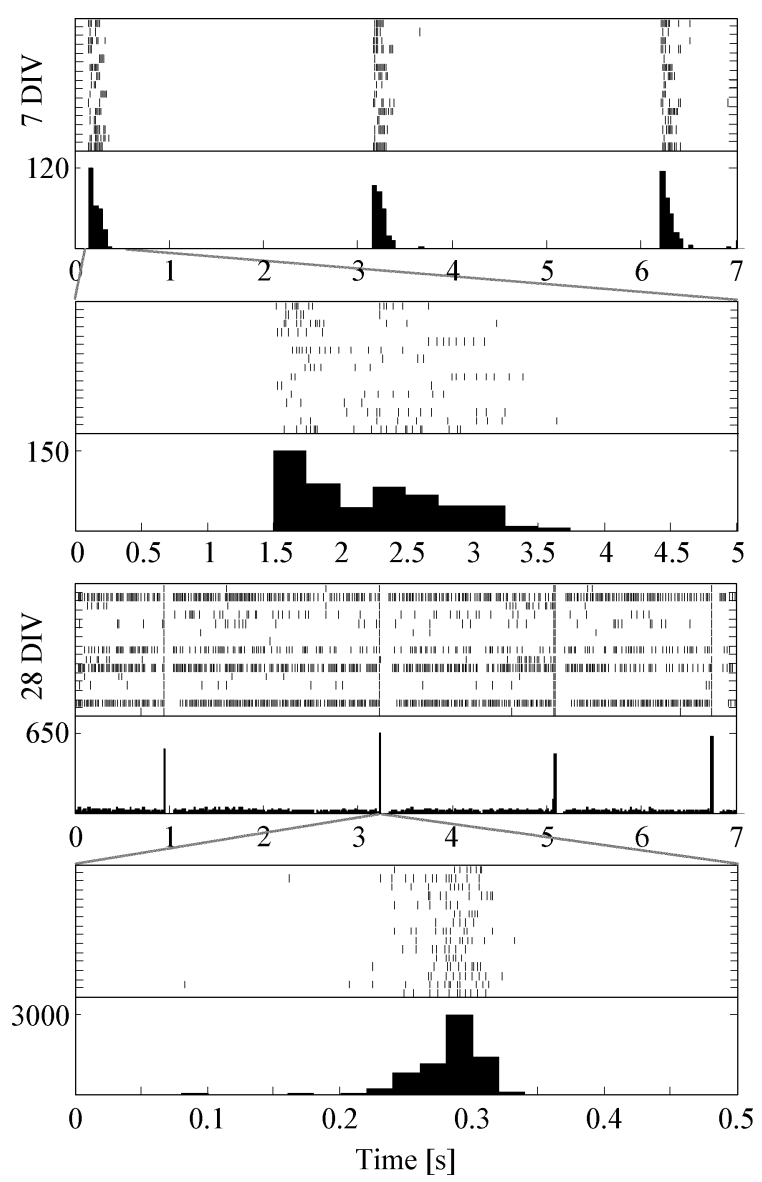

Fig. 3. Activity at two different stages of development (7, 28 DIV). The top traces show the spike times of 15 electrodes, the bottom traces show the AWSR in bins of $0.5,0.2,0.5,0.02 \mathrm{~s}$, respectively. At $7 \mathrm{DIV}$, bursts are wide, sometimes lasting several seconds. At 28 DIV, the bursts have shortened dramatically.

\section{RESULTS}

\section{A. General Progress and Burst Types}

The earliest electrical activity was recorded at 6 DIV and consisted of apparently uncorrelated activity on a small set of electrodes. Network bursts were regularly observed from about 7 DIV and were present for the entire observed period (max. 61 DIV). Early network bursts generally recruited few electrodes, lasted for seconds, and did not reach very high spike rates (Fig. 3).

At this stage in development, new sites were recruited; the network bursts shortened and became more intense. Around 9 DIV, the network bursts were intense enough to be automatically detected using the settings described in Section II and continued to be intense enough for the entire observed period. We checked for the influence of burst detection parameters and found that a large disparity existed between network bursts and false detections. We used the algorithm described by Segev et al. [35] to check whether there were discernible differences between bursts. In about one-third of all measurements, a second class was present with, on average, $15 \%$ bursts assigned to it. However, an evolving burst pattern may also introduce a second class. We have used the following rules to categorize our measurements (Table II): 1) There was one or a strong ( $>95 \%$ of bursts belonging to 1 class) dominant
TABLE II

Percentage of Measurements Assigned to One of Three Categories, Tabulated Per Culture. Note That There is an Obvious DifFerence Between Cultures. The Number of Measurements Per Culture is DENOTED BY N

\begin{tabular}{|r|r|r|r|r|}
\hline culture & $\mathrm{N}$ & Dominant (\%) & ordered (\%) & Unordered (\%) \\
\hline I & 59 & 98.31 & 1.69 & 0.00 \\
\hline II & 13 & 100.00 & 0.00 & 0.00 \\
\hline III & 15 & 53.33 & 26.67 & 20.00 \\
\hline IV & 19 & 78.95 & 10.53 & 10.53 \\
\hline V & 10 & 40.00 & 40.00 & 20.00 \\
\hline VI & 39 & 71.79 & 28.21 & 0.00 \\
\hline VII & 7 & 85.71 & 0.00 & 14.29 \\
\hline VIII & 8 & 50.00 & 37.50 & 12.50 \\
\hline IX & 7 & 71.43 & 28.57 & 0.00 \\
\hline X & 3 & 100.00 & 0.00 & 0.00 \\
\hline All & 180 & 80.00 & 15.00 & 5.00 \\
\hline
\end{tabular}

burst type. This was the case in $80 \%$ of all measurements. 2) There was a significant $(>5 \%$ and $>2$ bursts in the second class) second class, but the occurrences were in temporal order (suggesting a single, changing burst type). The degree of temporal ordering was quantified by calculating the number of alternations between bursts of the main class and second class. We compared this number to the theoretical value ( threshold $=$ mean-standard deviation, $\mu=N_{2} \cdot\left(N_{1}+N_{2}\right)^{-1}$, $\sigma=\mu \cdot\left(N_{1}+N_{2}\right)^{-0.5}$, where $N_{i}$ denotes the number of bursts assigned to class $i$ ) of a randomly ordered sequence of the same size. In $75 \%$ of the remaining measurements (i.e., $15 \%$ in total), a temporal ordering was found. 3) There was a significant ( $>5 \%$ and $>2$ bursts in the second class) second class without temporal ordering. When this is the case, there were (at least) two different burst patterns contributing significantly. We have encountered this situation in only $5 \%$ of all measurements. Considering the large quantity of measurements that fit in the first and second category, we interpreted our measurements as the development through time of a single burst pattern. We accepted the occasional presence of burst types expressed in parallel as a minor disturbance, as even in the $5 \%$ of measurements when this is the case, only $18 \%$ of bursts were assigned to a second class.

\section{B. Burst profiles}

Fig. 4 shows an example of all BPs during a 2-h measurement. BPs in the first hour (gray) are slightly wider than the profiles in the second hour of measurement (black). There was little variation in the general shape of the BPs from one burst to the other on a scale of $\sim 10 \mathrm{~ms}$. Also, the number of spikes, as indicated by the area underneath the graphs, and the peak firing rate appear to be well preserved over these $2 \mathrm{~h}$ of measurement. The correlation between individual bursts was extremely high, averaging 0.96 and a $10 \%$ lower boundary of 0.9 . In addition to this, we found only a small negative slope in the average correlation.

On a scale of days, Fig. 5 shows an example of the changes that BPs went through during maturation of the cultures. The BPs, averaged over $30 \mathrm{~min}$ for clarity, showed progressive small changes within a single measurement and larger changes between measurements. Several features of BPs in general are illustrated, such as a long tail, a distinct second mode, and differences in rising and falling phases. 

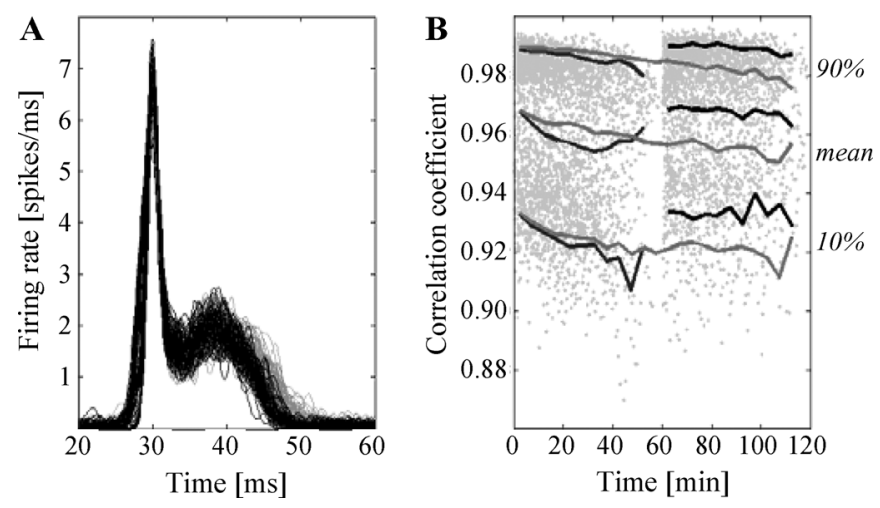

Fig. 4. (a) All BPs during a two-hour measurement at 14 DIV. Profiles from the first hour are shown in gray $(N=81)$, those from the second hour are shown in black $(N=74)$. Only the nonzero part is plotted. (b) Correlation coefficients between BPs shown in (a). The dots show individual correlation coefficients between BPs in the first hour $(0-60)$ and between bursts in the second hour (60-120). Lines show 5 min average, 10\% boundary, and $90 \%$ boundary. Average slope is $-0.007 / \mathrm{h}$.

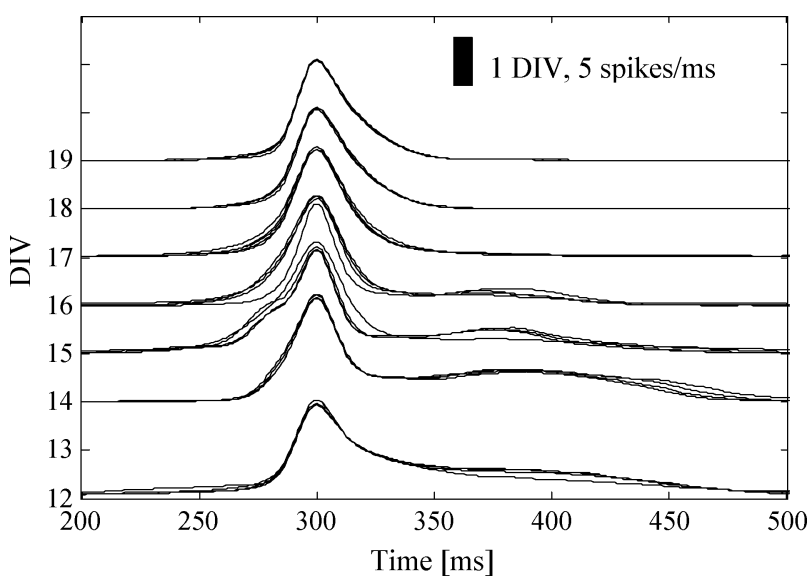

Fig. 5. Development of BPs over several days in vitro (DIV). One division is equal to one day in vitro (DIV) and also 5 spikes $/ \mathrm{ms}$.

We calculated the correlation coefficients between BPs for all cultures that we recorded from. For continuous recordings, we calculated the average correlation coefficient as a function of time difference binned in $15 \mathrm{~min}$. Fig. 6(a) displays the average of these intrameasurement correlations for all cultures. Similarly, the correlation coefficients between BPs measured on subsequent days (inter measurement correlations) are displayed in Fig. 6(b). We calculated the average decrease of correlation as a function of time for each culture and for all cultures in total. The influence of differences in the first day of measurement (9-12 DIV; 6b) was considered negligible.

\section{Phase Profiles}

A BP is essentially a global descriptive parameter and, thus, is unlikely to reveal changes between few pairs of electrodes. A phase profile can reveal more detailed information about individual electrodes. As Fig. 7 shows, the electrodes fired in a nonrandom order during a burst. Some were active as early as $100 \mathrm{~ms}$ before the main peak was reached, while others showed activity in the latter part of a burst. Most, if not all, active electrodes had a peak in their activity around the time of maximum
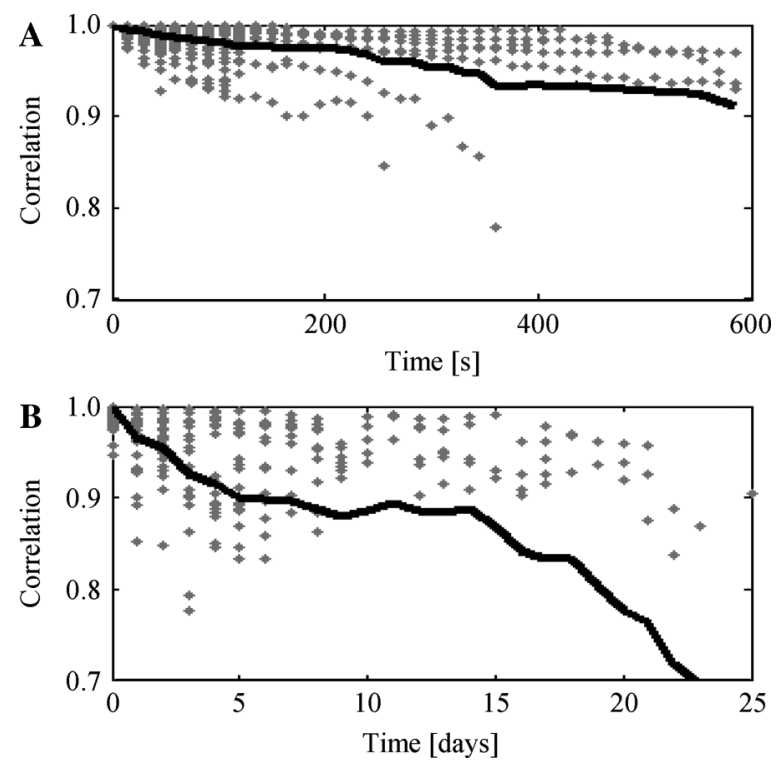

Fig. 6. Correlation coefficients between BPs. (a) Intrameasurement correlations. Each data point is a 15-min average per culture. (b) Intermeasurement correlations. Data points are average correlation coefficients between two sessions. Zero time indicates the first day of measurement and differs per culture (9-12 DIV). In both graphs, profiles were averaged over 15 min before calculation to decrease the computational load. The interpolating graphs are calculated by taking the mean slope per bin/day, with $(0,1)$ as the starting point.

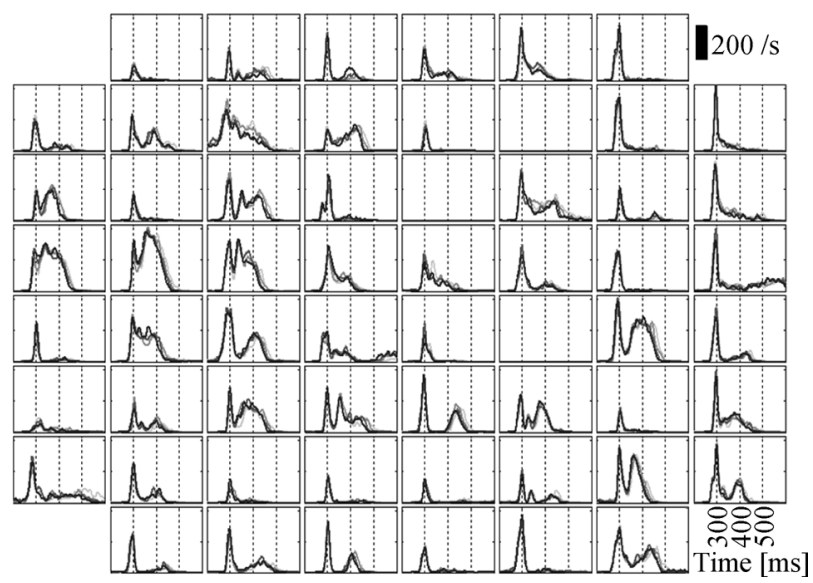

Fig. 7. Example of the stability of phase profiles for all electrodes in a 2-h measurement at 14 DIV (same data as in Fig. 4). Graph locations correspond to the MEA layout. Each graph shows four phase profiles, each of which is an average of more than $30 \mathrm{~min}(42,39,32$, and 32 bursts, respectively). The maximum AWSR is set at $300 \mathrm{~ms}$.

AWSR (i.e., $300 \mathrm{~ms}$ ). These observations are consistent with the observations already reported in [19] and [36]. In general, the profiles were too complex to be captured with a small number of descriptive parameters.

The PPs showed progressions that resemble those of the BPs. Fig. 8 shows PPs averaged per DIV, for several consecutive DIV. There was a clear trend, in BPs (corresponding BPs are shown in Fig. 5) and PPs, toward a single peak in the firing rate. A number of different progressive changes could be observed. For example, electrode 73 contributed very little in early recordings, but increased its number of elicited spikes during each measurement, eventually reaching a firing rate that was more than three times that found in early recordings. Electrode 14 started out 


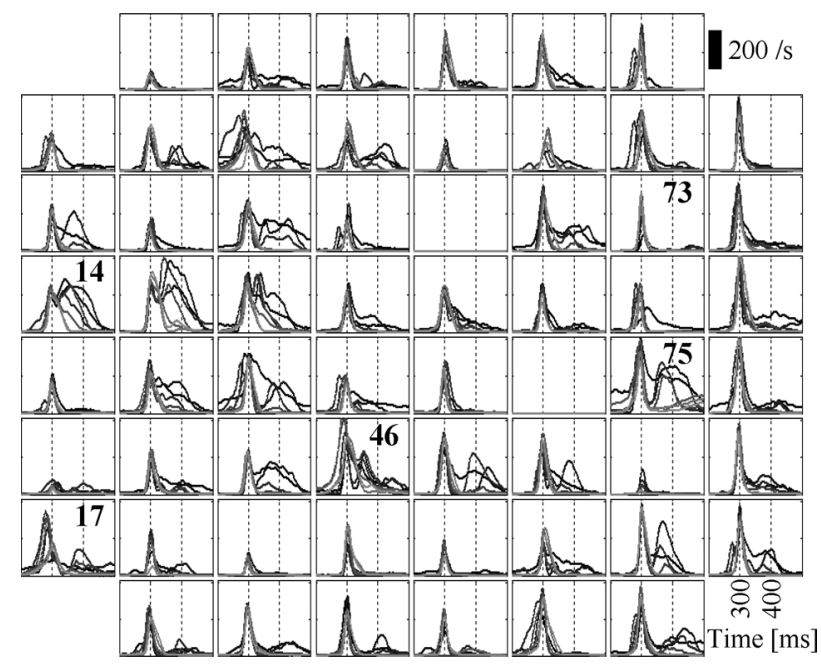

Fig. 8. Phase profiles of a single culture measured seven times in a span of eight days. The corresponding BPs are shown in Fig. 5. Graph locations correspond to the MEA layout. The AWSR peaks were set at $300 \mathrm{~ms}$.

with a dominant late phase of firing, which was completely absent in the late recordings. A bimodal firing pattern could be discerned in many electrodes (e.g., 75) at some time during the development of this culture. Electrode $17 \mathrm{had}$, in most measurements, a single mode of firing. The latency at which the maximum firing rate at electrode 17 was achieved changed during development. For most of the measurements, electrode 17 was early to fire.

The stability of PPs from burst to burst was less pronounced than that of BPs. Part of the difference could possibly be explained by the relatively small amount of spikes per electrode per burst. To compensate for this, we averaged PPs over 15 $\mathrm{min}$. The very small negative slope observed in the correlation coefficient between BPs indicated that bursts could be considered stable over such small periods (Fig. 4). It can be seen in Fig. 9 that correlation coefficients dropped at different rates, depending on the electrode. Correlation coefficients were generally higher, and had a lower slope, when the number of spikes elicited per electrode per burst was large. The inset in Fig. 9 shows that the correlation coefficients between BPs decrease with time approximately as a weighted average of the correlation coefficients between PPs. Some electrodes clearly showed an increased rate of change during some periods, while others showed a more constant rate of change. The latter indicated changes that affected the network as a whole; the first indicated that the role of a neuron within the network could change.

For comparison, the correlation coefficients of the culture shown in Fig. 8 are shown in Fig. 10. Here, also very different rates are observed, depending on electrode.

\section{Conditional Firing Probabilities}

Fig. 11 shows four examples of CFPs, as calculated by direct estimation [32] and by convolving PPs (Appendix). The convolution estimations of the CFPs follow the general shape of the direct estimate, often within the boundaries of one standard deviation. One limitation of the convolution estimate is that it cannot map refractory periods or discontinuities due to the continuous nature of the convolution and the smoothing applied

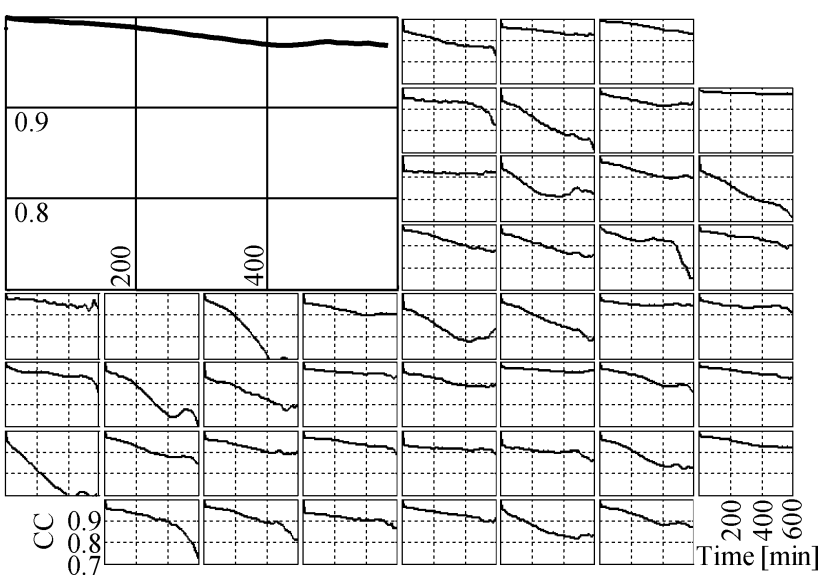

Fig. 9. Stability phase profiles within a 10-h continuous measurement. Phase profiles were averaged over $15 \mathrm{~min}$ before calculating correlations. Graph locations correspond to the MEA layout. The inset shows the correlation coefficients between BPs.

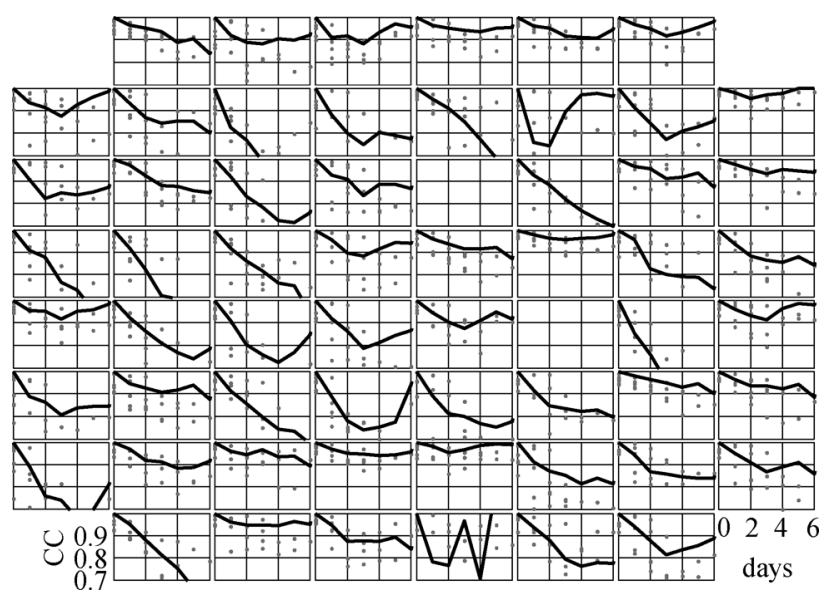

Fig. 10. Correlation coefficients as a function of time, calculated over several measurement sessions of a single culture. Same data as in Figs. 5, 7 and 8. The dots show individual correlation coefficients. The interpolating graphs are calculated by taking the mean slope per bin/day, with $(0.1)$ as the starting point.

when calculating the PPs. The estimation was more accurate when using small standard deviations in the filtering procedure of calculating the phase profiles.

\section{DISCUSSION}

At any time during the period that we measured, there was either one, or one strongly dominant type of burst expressed by the culture. This contradicts several other findings, which reported a large diversity in bursts [15], [29], [35]. One difference is that we use a chemically defined medium [19], [33], whereas groups observing burst diversity all use $5 \%$ horse serum in their culture medium [15], [35]. Promoting glial cells this way has a noticeable influence on the network activity [37], [38], and may well lead to differences in bursting behavior. The apparent discrepancy may also be explained by differences in culture age. We measured cultures predominantly in the maturation phase, up to 21 DIV [3], [5], [14], [19], [23], [39], while other studies report on cultures which were considered mature, beyond 21 DIV.

The apparent absence of diversity in bursts may have a profound impact on the information processing capabilities of a net- 

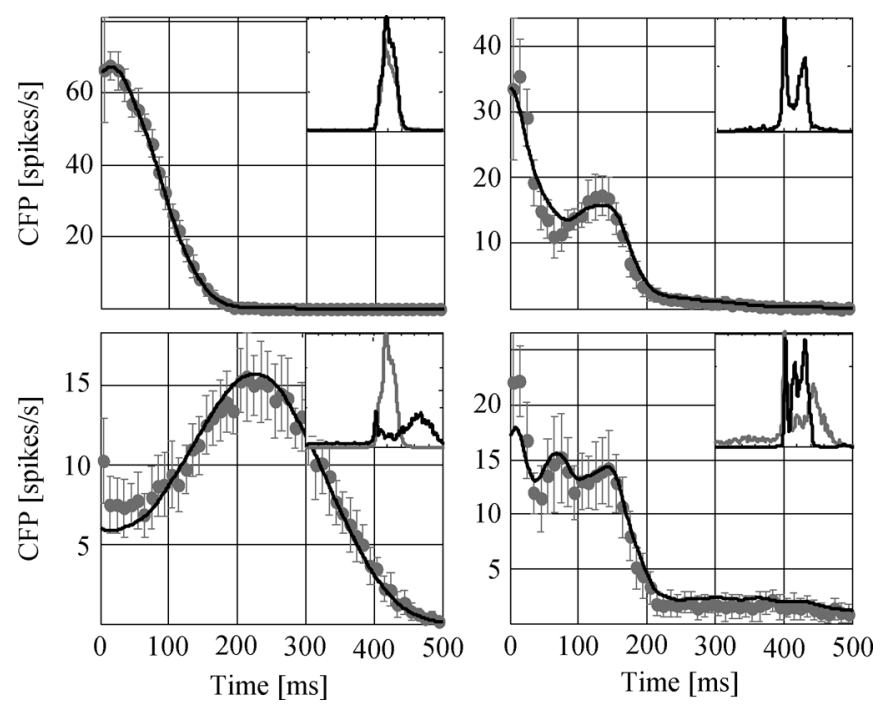

Fig. 11. Examples of conditional firing probabilities (CFPs) calculated by the direct method and the convolution method, based on $1 \mathrm{~h}$ of data measured at 14 DIV. Filled circles and standard deviations show the CFP calculated by (3), lines show the result of convolving the phase profiles. The insets show the phase profiles of the electrodes under consideration from $500 \mathrm{~ms}$ before to $500 \mathrm{~ms}$ after the burst peak.

work. Beggs and Plenz [29] conclude from brain slice recordings that the diversity of bursts is such that, should bursts be used to process information, the transfer of information is optimal. In this respect, our cultures would be unable to process any information, at least during the maturation phase. Since the cultures are randomly plated and have not received any external (electrical) stimuli since plating, one can assume that structure and activity have been shaped using self-generated signals [18]. Thus, the lack of structure and inputs may well contribute to the absence of burst diversity in the cultures during the maturation phase.

The observation that BPs show very little variation from one burst to the next, and change their shape over a time scale of several hours has previously been made by Van Pelt et al. [19], but was never quantified. The correlations coefficients between bursts and those between PPs show that the variation in profile shape is very low. Some variation between bursts is reduced by Gaussian filtering, but the standard deviation used $(5 \mathrm{~ms})$ is very small compared to the length of a burst and the relevant features of the burst are still captured. The correlation coefficient as a measure to compare profiles is certainly biased to some extent. The correlation coefficient is more sensitive to changes in the main peak, where the firing rate is high, and less sensitive to any of the smaller, but nevertheless important, features. Bias is noticeable because many changes exclude the main peak, and their influence on the correlation coefficient depends on the size of the changed features relative to the peak size.

Both BPs and PPs alter shape in an incremental way. This suggests that plasticity mechanisms are responsible for these changes, rather than random variations. Apart from synaptogenesis, which occurs mainly up to 18 DIV, modifications of synaptic efficacy occur when connected neurons spike within 20 ms of each other [40]. During network bursts, most electrodes spike in a period of about $100 \mathrm{~ms}$ and, thus, massive reinforcements of synaptic efficacies can be expected. These reinforcements help to preserve burst structure when there are no large changes in input and/or structure. It is known that during the third week in vitro, there is a period in which large numbers of synapses are pruned [41], [42]. In [19], large changes in BPs are observed around this period, only to stabilize again afterwards. We have not observed changes of such magnitude, possibly due to less and/or slower pruning of synapses.

We found that CFPs can be estimated from PPs via convolution which, combined with the stability found by both methods, suggests that functional connectivity and burst structure are expressions of the same underlying physical network structure. In view of multiple burst types reported by several researchers [15], [29], [35], it is necessary to first discriminate between different types of bursts (e.g., using the method described in [35]), and then calculate the PPs and CFPs, such that functional connectivity for each burst type is revealed.

\section{APPENDIX}

The phase profiles (PPs) can be used to construct electrode-to-electrode relationships by considering all combinations of two PPs and convolving these. The PPs are firing probabilities of the stereotypical activity during bursts. We will show that convolving two PPs yields the CFP of one electrode to another. These CFPs, in turn, define a network of (generalized or functional) connections in terms of strength and latency. We start with deriving the CFP, which describes the probability that neuron $j$ fires at time $t+\tau$ when neuron $i$ fires at $t$. We can describe the two spike trains by a summation of Dirac pulses (Fig. 2)

$$
\begin{aligned}
& v_{i}(t)=\sum_{k=1}^{K} \delta\left(t-t_{k}\right) \\
& v_{j}(t)=\sum_{m=1}^{M} \delta\left(t-t_{m}\right)
\end{aligned}
$$

where $t_{k}$ denotes the time at which neuron $i$ fires its $k^{\text {th }}$ action potential and similarly for neuron $j$. The CFP counts all occurrences that $t_{m}-t_{k}$ equals a certain delay $\tau$, divided by the number of action potentials $K$ elicited by neuron $i$

$$
\mathrm{CFP}_{i j}(\tau)=\frac{1}{K} \sum_{k=1}^{K} \sum_{m=1}^{M} \delta\left(\tau-\left(t_{m}-t_{k}\right)\right)
$$

Formula (5) is the direct estimate of the CFP, and is implemented in discrete form by Le Feber et al. [32]. The estimation accuracy increases when the number of action potentials in both time series $(K$ and $M)$ is large. It also implies that the relation between $i$ and $j$ does not change with time, and that both time series are stationary.

In a culture that exhibits bursting, a large fraction of the action potentials occurs during network bursts (it is regularly observed that $80 \%$ of spikes are within bursts). The stereotypical firing pattern during bursts is described by the PPs. With proper 
scaling, the phase profiles can be interpreted as probability density functions (PDFs) of eliciting a single spike during a burst. In other words, $t_{k}$ is a random variable with a distribution function $f_{i}(t)$ given by the normalized PP of neuron $i$. The conditional probability density function is given by

$$
f_{j \mid i}(\tau)=\int_{t_{k}} \int_{t_{m}} \delta\left(\tau-t_{m}+t_{k}\right) f_{i, j}\left(t_{k}, t_{m}\right) d t_{k} d t_{m} .
$$

We will approximate the joint probability density function $f_{i, j}$ by

$$
f_{i, j}\left(t_{k}, t_{m}\right) \cong f_{i}\left(t_{k}\right) f_{j}\left(t_{m}\right) .
$$

This approximation is valid for the case where each neuron elicits one spike. Multiple spikes may be elicited on $j$ in response to a single spike in $i$ (all during bursts). This is accounted for by a scaling factor $J(\approx M / K)$, thereby implicitly assuming that spikes elicited by $j$ are randomly drawn from $f_{j}$. The CFP can then be expressed as

$$
\begin{aligned}
\operatorname{CFP}_{i j}(\tau) & =J \cdot f_{j \mid i}(\tau) \\
& =J \int_{t_{k}} \int_{t_{m}} \delta\left(\tau-t_{m}+t_{k}\right) f_{i}\left(t_{k}\right) f_{j}\left(t_{m}\right) d t_{k} d t_{m} .
\end{aligned}
$$

One integral can now be eliminated using the sifting property of the Dirac function

$$
\mathrm{CFP}_{i j}(\tau)=J \int_{t_{m}} f_{i}\left(t_{m}-\tau\right) f_{j}\left(t_{m}\right) d t_{m} .
$$

Equation (9) constitutes a convolution between the phase profiles of electrodes $i$ and $j$. The convolution estimate of the CFP in (9) and the direct estimation in (5) yield similar curves (Fig. 11). The main difference is that the direct method uses mainly averaging over time, while the convolution method uses interpolation (Gaussian filtering) as well as averaging over bursts (i.e., time). Consider also that not all action potentials occur in bursts; we expect that the CFP is better estimated directly from its definition. However, PPs are primarily meant to show contributions of single electrodes to network bursts.

\section{ACKNOWLEDGMENT}

The authors would like to thank R. Wiertz for his work on the preparation and maintenance of cultures.

\section{REFERENCES}

[1] M. Giugliano, P. Darbon, M. Arsiero, H. R. Luscher, and J. Streit, "Single-neuron discharge properties and network activity in dissociated cultures of neocortex," J. Neurophysiol., vol. 92, pp. 977-996, 2004.

[2] A. Rauch, G. La Camera, H. R. Luscher, W. Senn, and S. Fusi, "Neocortical pyramidal cells respond as integrate-and-fire neurons to in vivo-like input currents," J. Neurophysiol., vol. 90, pp. 1598-1612, 2003.

[3] G. Shahaf and S. Marom, "Learning in networks of cortical neurons," J. Neurosci., vol. 21, pp. 8782-8788, 2001.

[4] S. Marom and D. Eytan, "Learning in ex-vivo developing networks of cortical neurons," Progr. Brain Res., vol. 147, pp. 189-199, 2005.
[5] S. Marom and G. Shahaf, "Development, learning and memory in large random networks of cortical neurons: Lessons beyond anatomy," $Q$. Rev. Biophys., vol. 35, pp. 63-87, 2002.

[6] D. Eytan, N. Brenner, and S. Marom, "Selective adaptation in networks of cortical neurons," J. Neurosci., vol. 23, pp. 9349-9356, 2003.

[7] M. E. Ruaro, P. Bonifazi, and V. Torre, "Toward the neurocomputer: Image processing and pattern recognition with neuronal cultures," IEEE Trans. Biomed. Eng., vol. 52, no. 3, pp. 371-383, Mar. 2005.

[8] E. Maeda, Y. Kuroda, H. P. Robinson, and A. Kawana, "Modification of parallel activity elicited by propagating bursts in developing networks of rat cortical neurones," Eur.J Neurosci., vol. 10, pp. 488-496, 1998.

[9] D. A. Wagenaar, J. Pine, and S. M. Potter, "Searching for plasticity in dissociated cortical cultures on multi-electrode arrays," J .Negative Results Biomedic., vol. , 2006.

[10] Y. Jimbo, H. P. Robinson, and A. Kawana, "Strengthening of synchronized activity by tetanic stimulation in cortical cultures: Application of planar electrode arrays," IEEE Trans Biomed. Eng, vol. 45, no. 11, pp. 1297-1304, Nov. 1998.

[11] Y. Jimbo, T. Tateno, and H. P. C. Robinson, "Simultaneous induction of pathway specific potetiation and depression in networks of cortical neurons," Biophys. J., vol. 76, pp. 670-678, 1999.

[12] R. Madhavan, Z. C. Chao, and S. M. Potter, "Spontaneous bursts are better indicators of tetanus-induced plasticity than responses to probe stimuli," presented at the 2nd Int. IEEE EMBS Conf. Neural Engineering, Arlington, VA, 2005.

[13] Z. C. Chao, D. J. Bakkum, D. A. Wagenaar, and S. M. Potter, "Effects of random external background stimulation on network synaptic stability after tetanization," Neuroinform., pp. 263-280, 2005.

[14] J. Van Pelt, M. A. Corner, P. S. Wolters, W. L. Rutten, and G. J. Ramakers, "Longterm stability and developmental changes in spontaneous network burst firing patterns in dissociated rat cerebral cortex cell cultures on multielectrode arrays," Neurosci. Lett., vol. 361, pp. 86-89, 2004.

[15] D. A. Wagenaar, R. Madhavan, J. Pine, and S. M. Potter, "Controlling bursting in cortical cultures with closed-loop multi-electrode stimulation," J Neurosciol., vol. 25, pp. 680-688, 2005.

[16] M. Chiappalone, M. Bove, A. Vato, M. Tedesco, and S. Martinoia, "Dissociated cortical networks show spontaneously correlated activity patterns during in vitro development," Brain Res., vol. 1093, pp. 41-53, 2006.

[17] Y. Ikegaya, G. Aaron, R. Cossart, D. Aronov, I. Lampl, D. Ferster, and R. Yuste, "Synfire chains and cortical songs: Temporal modules of cortical activity," Sci., vol. 304, pp. 559-564, 2004.

[18] J. van Pelt, I. Vajda, P. S. Wolters, M. A. Corner, and G. J. Ramakers, "Dynamics and plasticity in developing neuronal networks in vitro," Progr. Brain Res., vol. 147, pp. 173-188, 2005.

[19] J. van Pelt, P. S. Wolters, M. A. Corner, W. L. Rutten, and G. J. Ramakers, "Long-term characterization of firing dynamics of spontaneous bursts in cultured neural networks," IEEE Trans Biomed. Eng., vol. 51, no. 11, pp. 2051-2062, Nov. 2004.

[20] D. A. Wagenaar, J. Pine, and S. M. Potter, "An extremely rich repertoire of bursting patterns during the development of cortical cultures," $B M C$ Neurosci., vol. 7, p. 11, 2006.

[21] M. A. Corner, J. van Pelt, P. S. Wolters, R. E. Baker, and R. H. Nuytinck, "Physiological effects of sustained blockade of excitatory synaptic transmission on spontaneously active developing neuronal networks-An inquiry into the reciprocal linkage between intrinsic biorhythms and neuroplasticity in early ontogeny," Neurosci. Biobehav. Rev., vol. 26, pp. 127-185, 2002.

[22] M. Ichikawa, K. Muramoto, K. Kobayashi, M. Kawahara, and Y. Kuroda, "Formation and maturation of synapses in primary cultures of rat cerebral cortical cells: An electron microscopic study," Neurosci Res., vol. 16, pp. 95-103, 1993.

[23] H. Kamioka, E. Maeda, Y. Jimbo, H. P. Robinson, and A. Kawana "Spontaneous periodic synchronized bursting during formation of mature patterns of connections in cortical cultures," Neurosci Lett., vol. 206, pp. 109-112, 1996.

[24] G. Kiddie, D. McLean, A. Van Ooyen, and B. Graham, "Biologically plausible models of neurite outgrowth," Progr. Brain Res., vol. 147, pp. 67-80, 2005.

[25] H. Neuhoff, J. Roeper, and M. Schweizer, "Activity-dependent formation of perforated synapses in cultured hippocampal neurons," Eur. J. Neurosci., vol. 11, pp. 4241-4250, 1999. 
[26] A. van Ooyen and J. van Pelt, "Activity-dependent neurite outgrowth and neural network development," Progr. Brain Res., vol. 102, pp. 245-259, 1994.

[27] A. Van Ooyen, J. Van Pelt, and M. A. Corner, "Implications of activity dependent neurite outgrowth for neuronal morphology and network development," J. Theor. Biol., vol. 172, pp. 63-82, 1995.

[28] C. van Oss and A. van Ooyen, "Effects of inhibition on neural network development through activity-dependent neurite outgrowth," J. Theor. Biol., vol. 185, pp. 263-280, 1997.

[29] J. M. Beggs and D. Plenz, "Neuronal avalanches are diverse and precise activity patterns that are stable for many hours in cortical slice cultures," J. Neurosci., vol. 24, pp. 5216-5229, 2004.

[30] I. Baruchi and E. Ben-Jacob, "Functional holography of recorded neuronal networks activity," Neuroinform., vol. 2, pp. 333-352, 2004.

[31] W. J. Melssen and W. J. Epping, "Detection and estimation of neural connectivity based on crosscorrelation analysis," Biol. Cybern., vol. 57, pp. 403-414, 1987.

[32] J. Le Feber, W. L. C. Rutten, J. Stegenga, P. S. Wolters, G. J. A. Ramakers, and J. van Pelt, "Conditional firing probabilities in cultured neuronal networks: A stable underlying structure in widely varying spontaneous patterns," J. Neural Eng., vol. 4, pp. 54-67, 2007.

[33] H. J. Romijn, F. van Huizen, and P. S. Wolters, "Towards an improved serum-free, chemically defined medium for long-term culturing of cerebral cortex tissue," Neurosci. Biobehav. Rev., vol. 8, pp. 301-334, 1984.

[34] D. A. Wagenaar, T. B. DeMarse, and S. M. Potter, "MeaBench: A toolset for multi-electrode data acquisition and online analysis," presented at the 2nd International IEEE EMBS Conference on Neural Engineering, Arlington, VA, 2005.

[35] R. Segev, I. Baruchi, E. Hulata, and E. Ben-Jacob, "Hidden neuronal correlations in cultured networks," Phys. Rev. Lett., vol. 92, p. 118102, 2004.

[36] D. Eytan and S. Marom, "Dynamics and effective topology underlying synchronization in networks of cortical neurons," J. Neurosci., vol. 26, pp. 8465-8476, 2006

[37] G. J. Brewer, M. D. Boehler, and B. C. Wheeler, "Added astroglia promote greater synapse density and higher activity," presented at the 5th Int. Meeting on Substrate-Integrated Micro Electrode Arrays, Reutlingen, Germany, 2006.

[38] Y. Nam, J. Chang, D. Khatami, G. J. Brewer, and B. C. Wheeler, "Patterning to enhance activity of cultured neuronal networks," Proc. Inst. Elect. Eng., Nanobiotechnol., vol. 151, pp. 109-115, 2004.

[39] S. Watanabe, Y. Jimbo, H. Kamioka, Y. Kirino, and A. Kawana, "Development of low magnesium-induced spontaneous synchronized bursting and GABAergic modulation in cultured rat neocortical neurons," Neurosci. Lett., vol. 210, pp. 41-44, 1996.

[40] L. I. Zhang, H. W. Tao, C. E. Holt, W. A. Harris, and M. Poo, "A critical window for cooperation and competition among developing retinotectal synapses," Nature, vol. 395, pp. 37-44, 1998.

[41] G. J. Ramakers, H. van Galen, M. G. Feenstra, M. A. Corner, and G. J. Boer, "Activity-dependent plasticity of inhibitory and excitatory amino acid transmitter systems in cultured rat cerebral cortex," Int. J. Dev Neurosci., vol. 12, pp. 611-621, 1994.

[42] F. van Huizen, H. J. Romijn, and A. M. Habets, "Synaptogenesis in rat cerebral cortex cultures is affected during chronic blockade of spontaneous bioelectric activity by tetrodotoxin," Brain Res., vol. 351, pp. 67-80, 1985.

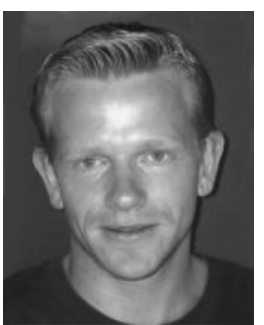

Jan Stegenga was born in Wijckel, The Netherlands, on April 19, 1979. He received the M.Sc. degree in electrical engineering from the University of Twente, Enschede, The Netherlands, in 2004, where he is currently pursuing the $\mathrm{Ph} . \mathrm{D}$. degree.

Currently, he researches memory and learning in cultured neuronal networks.

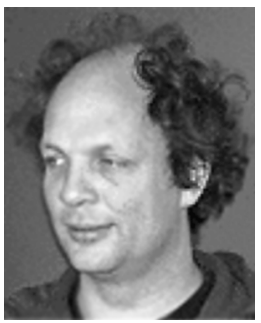

Joost Le Feber was born in Veldhoven, the Netherlands, in 1967. He received the M.Sc. degree in electrical engineering from the Delft University of Technology, Delft, The Netherlands, in 1991 and the Ph.D. degree on quantitative models that relate autonomic nerve activity to mechanical variables in the lower urinary tract from Erasmus University, Rotterdam, the Netherlands, in 2000.

After a four-year Postdoctoral Fellowship at Groningen University, Groningen, The Netherlands, where he worked in the field of artificial intelligence, he is now with the University of Twente, Enschede, The Netherlands. His research interests include learning and memory, cultured neuronal networks, and computational neuroscience.

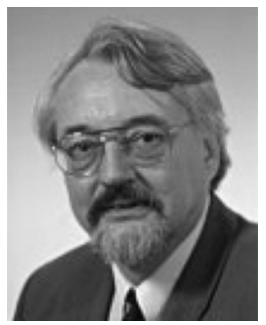

Enrico Marani was born in 1946. He received the $\mathrm{Ph} . \mathrm{D}$. degree on the structure and function of the cerebellum from Leiden University, Leiden, The Netherlands, in 1982 .

In 1986, he became Head of the Neuroregulation Group at the Leiden University Medical Center, Leiden. He has studied regeneration of the central the nervous system since 1990. He was Editor of Archives of Physiology and Biochemistry, Advances in Anatomy, Embryology and Cell Biology, and Co-editor of BioMedical Reviews. His research interests focus on the application of neurotechnology in medicine.

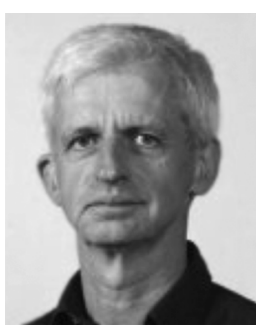

Wim L. C. Rutten was born in 1950. He received the Ph.D. degree in solid state physics from Leiden University, Leiden, The Netherlands, in 1979

$\mathrm{He}$ is the Chair of Neurotechnology with the Faculty of Electrical Engineering, Mathematics and Computer Science and with the Institute for BioMedical Technology (BMTI) of the University of Twente, Enschede, The Netherlands. He was trained as an Experimental Physicist with Leiden University. He was a Physicist-Audiologist with Leiden University Hospital in hearing science and electrophysiology until 1985, before joining the Biomedical Engineering Group at the University of Twente, Enschede. He is the Leader of the Neural and Cellular Engineering Program of BMTI, with a focus on live learning neural networks, the development of a cultured probe, and on brain-computer interfaces. 